\title{
Instability and trade in currency areas
}

\author{
Alberto Alonso ${ }^{\mathrm{a}, 1}$, Luis C. Corchón ${ }^{\mathrm{b}, *}$, Vanesa Guzmán ${ }^{\mathrm{c}, 2}$ \\ a Department of Applied Economics III, Universidad Complutense de Madrid, Campus de Somosaguas, 28223 Madrid, Spain \\ ${ }^{\mathrm{b}}$ Department of Economics, Universidad Carlos III de Madrid, 126 Madrid Road, Getafe, 28903 Madrid, Spain \\ ${ }^{\mathrm{c}}$ Department of Economics, Universidad San Pablo-CEU, 23 Julián Romea Road, 28003 Madrid, Spain
}

Received 15 October 2004; accepted 23 June 2006

Available online 13 November 2006

\begin{abstract}
In a currency area, when a country faces a positive shock inflation goes up, real interest rate decreases and competitiveness deteriorates. We show that the stability of equilibrium depends on the rationality of expectations and budget balance of the public sector.
\end{abstract}

(c) 2006 Elsevier B.V. All rights reserved.

Keywords: Currency areas; Trade; Real interest rate; Common monetary policy; National fiscal policies

JEL classification: F15; E63

\section{Introduction}

In a currency area, compensating the effect of an idiosyncratic shock on a member country by adjusting the exchange rate is no longer an option. The literature recognizes several factors that may take the role of exchange rate: factor mobility (Mundell, 1961), price/wage flexibility (Friedman, 1953; Kawai, 1987), fiscal federalism (Kenen, 1969) and trade among member countries (McKinnon, 1963).

\footnotetext{
is This paper was presented at the Economic Theory Seminar in Malaga. We acknowledge comments from the audience and Olivier Blanchard and financial support from CAYCIT, BEC 2002-02194. We are solely responsible for any error or misunderstanding.

* Corresponding author. Tel.: +34 91 6249617; fax: +34 916249329.

E-mail addresses: alberto@ccee.ucm.es (A. Alonso), lcorchon@eco.uc3m.es (L.C. Corchón), vanguz@telefonica.net (V. Guzmán).

1 Tel.: +3491 3942580; fax: +34913942582.

2 Tel.: +34922 150591.
} 
The latter effect arises either from income or inflation rate differentials. ${ }^{3}$ In this note we concentrate on the second effect. The conjecture is that such an effect stabilizes the economies in the currency area because if a country experiences, say, a positive shock, its inflation goes up, decreasing the competitiveness of this country and neutralizing the initial effect (Alesina et al., 2001, 18-19). We call this the competitive effect.

However, when the rate of inflation goes up, the real interest rate decreases. This effect goes in the opposite direction to the competitive effect because it stimulates the economy of the country that has experienced the positive shock. We call this the real interest effect (this effect is the basis of the so-called "Walters critique" to the EMS).

In this note we provide a simple model to study the working of both effects that bears some resemblance to a model of Tobin (1975) for a closed economy. We assume two countries and that all relevant functions are linear. We also assume that the only difference between countries is the size of potential output. Public expenditure is, either constant or tuned in order to offset inflation. The central bank only cares about maintaining a constant average inflation. We show that if the central bank attains its target, the nominal interest rate is constant (Lemma 1).

We identify a range of parameters for which equilibrium is stable (Proposition 1). It turns out that without an activist fiscal policy, these values are not very reasonable. In particular, expectations in equilibrium cannot be rational. This is due to the fact that the competitive effect does not work if agents anticipate correctly inflation because in this case supply becomes totally inelastic. Moreover, a country receiving a positive shock has a rising inflation, but not necessarily a relatively high inflation. ${ }^{4}$

\section{The model}

Time is continuous. All variables are twice continuously differentiable with respect to time. A dot above a variable represents its derivative with respect to time. Two dots indicate a second derivative.

There are two countries denoted by $j=1,2$. Let $Y_{n}^{j}$ be the potential income of country $j$. Let $\lambda$ and $1-\lambda$ be the relative size of the potential income of countries 1 and 2, i.e.

$$
(1-\lambda) Y_{1}^{n}=\lambda Y_{2}^{n}
$$

Countries are identical except for the scale factor $\lambda$. The income of $j, Y^{j}$, is the sum of consumption, investment, public expenditure, $G^{j}$, and net exports, $S^{j}$. Investment is linear on the real interest rate, $r^{j}$. Consumption is linear on income net of taxes. Taxes are linear on income. Thus

$$
Y^{j}=F_{j}-B_{j} r^{j}+C_{j} S^{j}+D_{j} G^{j}
$$

where $F_{j}, B_{j}, C_{j}, D_{j}>0$. This is the usual IS curve. Taking units, $D_{1}=D_{2}=1$. Since countries are identical except for the scale factor, $C_{1}=C_{2}=C$, and the relative impact of the interest rate on output equals the

\footnotetext{
3 The early literature focussed on price levels, not on inflation rates.

4 If inflation is rising in a country, eventually, it will catch up to the inflation in the other country. However, at this point, potential output is different from real output (except in exceptional cases). Therefore the equality between inflation rates will not persist, producing a cycle. See our comments at the end of Section 2.
} 
relative size of this country, $(1-\lambda) B_{1}=\lambda B_{2}$. If $i$ is the nominal interest rate and $\Pi_{\mathrm{e}}^{j}$ the expected rate of inflation of country $j, i \equiv r^{j}+\Pi_{\mathrm{e}}^{j}$. Expectations are formed according to

$$
\Pi_{\mathrm{e}}^{j}=a \Pi^{j}-b \dot{\Pi}^{j}
$$

where $a>0$. Thus, if inflation rate is constant and $a=1$ we have perfect foresight. From Eq. (3) we get $\dot{\Pi}_{\mathrm{e}}^{j}=a \dot{\Pi}^{j}-b \ddot{\Pi}^{j}$.

The supply side is modelled by a Phillips curve with expectations, i.e.

$$
\Pi^{j}=\bar{\beta}_{j}\left(Y^{j}-Y_{n}^{j}\right)+\Pi_{\mathrm{e}}^{j}
$$

where $\bar{\beta}_{j} \equiv \beta / Y_{n}^{j}$.

Notice that $\bar{\beta}_{j}\left(Y_{n}^{j}-Y^{j}\right)=\Pi_{\mathrm{e}}^{j}-\Pi^{j}=(a-1) \Pi^{j}-b \dot{\Pi}^{j}$. Therefore if $a \approx 1, b<0$ is absurd because it implies that when actual output is larger than potential output, inflation rate is decreasing. Thus, from now on we assume $b>0$.

Net exports are assumed to be

$$
\dot{S}^{1}=Q \operatorname{Ln}\left(\frac{P^{2}}{P^{1}}\right)^{\alpha}
$$

where $Q>0, \alpha>0$ and $P^{j}$ is the price in $j$. Clearly, $S^{1}=-S^{2}$. When prices are identical in both countries, $S^{1}=0$. Setting $K \equiv Q \alpha$ we obtain

$$
\stackrel{\bullet}{S}=K\left(\Pi^{2}-\Pi^{1}\right)
$$

The fiscal authority of each country implements a fiscal policy to stabilize the national economy such that the variation in public expenditure is linear on the rate of change of inflation. ${ }^{5}$

$$
\stackrel{\bullet j}{G}=-\delta_{j} \stackrel{\bullet}{\Pi}
$$

where $\delta_{j} \geq 0$ and $(1-\lambda) \delta_{1}=\lambda \delta_{2}$.

Finally, we assume that the central bank stabilizes weighted average inflation, $\bar{\Pi}$, defined as $\bar{\Pi} \equiv \lambda \Pi^{1}+(1-\lambda) \Pi^{2}$.

Lemma. If average inflation is constant, the nominal interest rate is constant.

Proof. Plugging Eqs. (2) and (4) in the definition of $\bar{\Pi}$ :

$$
\begin{aligned}
\bar{\Pi}= & \lambda\left\{\bar{\beta}_{1}\left[F_{1}-B_{1}\left(i-\Pi_{\mathrm{e}}^{1}\right)+C S^{1}+G^{1}-Y_{1}^{n}\right]+\Pi_{\mathrm{e}}^{1}\right\} \\
& +(1-\lambda)\left\{\bar{\beta}_{2}\left[F_{2}-B_{2}\left(i-\Pi_{\mathrm{e}}^{2}\right)-C S^{1}+G^{2}-Y_{2}^{n}\right]+\Pi_{\mathrm{e}}^{2}\right\}
\end{aligned}
$$

Differentiating with respect to time and rearranging:

$$
\dot{\bullet}=\frac{\stackrel{\bullet}{\Pi}_{\mathrm{e}}\left[\lambda\left(\bar{\beta}_{1} B_{1}+1\right)\right]+\dot{\Pi}_{\mathrm{e}}^{2}\left[(1-\lambda)\left(\bar{\beta}_{2} B_{2}+1\right)\right]+C \dot{S}^{1}\left[\lambda \bar{\beta}_{1}-(1-\lambda) \bar{\beta}_{2}\right]+\lambda \bar{\beta}_{1} \dot{G}^{1}+(1-\lambda) \bar{\beta}_{2} \dot{G}^{2}}{\lambda \bar{\beta}_{1} B_{1}+(1-\lambda) \bar{\beta}_{2} B_{2}}
$$

\footnotetext{
${ }^{5}$ For simplicity, we disregard boundary problems associated with the non-negativity of public expenditure.
} 
Using Eqs. (3), (5) and (6)

$$
\dot{i}=\frac{\stackrel{\bullet}{\Pi}^{1} a \lambda\left[\bar{\beta}_{1}\left(B_{1}-\delta_{1}\right)-\bar{\beta}_{2}\left(B_{2}-\delta_{2}\right)\right]-\ddot{\Pi}^{1} b \lambda\left(\bar{\beta}_{1} B_{1}-\bar{\beta}_{2} B_{2}\right)+K\left[\bar{\Pi}-\Pi^{1} / 1-\lambda\right] C\left[\lambda \bar{\beta}_{1}-(1-\lambda) \bar{\beta}_{2}\right]}{\lambda \bar{\beta}_{1} B_{1}+(1-\lambda) \bar{\beta}_{2} B_{2}}
$$

Thus, under our assumptions, $\dot{i}=0$.

From now on we assume that the central bank achieves its objective. Plugging Eqs. (2) and (3) in Eq. (4), for $j=1$

$$
\ddot{\Pi}^{1}\left(\bar{\beta}_{1} B_{1}+1\right) b=\Pi^{1}\left[\left(\bar{\beta}_{1} B_{1}+1\right) a-1\right]+\bar{\beta}_{1}\left(F_{1}-B_{1} i+C S^{1}+G^{1}-Y_{1}^{n}\right)
$$

Differentiating with respect to time using Eqs. (5), (6) and the definition of $\bar{\Pi}$,

$$
\begin{aligned}
& \ddot{\Pi}^{1}\left(\bar{\beta}_{1} B_{1}+1\right) b=\dot{\Pi}^{1}\left[\left(\bar{\beta}_{1} B_{1}+1\right) a-\bar{\beta}_{1} \delta_{1}-1\right]+\bar{\beta}_{1} C K \frac{\left(\bar{\Pi}-\Pi^{1}\right)}{1-\lambda} \\
& \ddot{\Pi}^{2}=-\frac{\lambda}{1-\lambda} \stackrel{\bullet !}{\Pi}^{1}
\end{aligned}
$$

If Eq. (7) is solved, Eq. (8) is solved too. Therefore let us solve Eq. (7).

Proposition 1. If $a<1+\bar{\beta}_{1} \delta_{1} / \bar{\beta}_{1} B_{1}+1$ and the competitive effect is large enough, the trajectory of the rate of inflation in country 1 is stable.

Proof. Eq. (7) can be written as $\ddot{\Pi}^{1}+d_{1} \stackrel{\bullet}{\Pi}^{1}+d_{2} \Pi^{1}=d_{2} \bar{\Pi}$, where and

$$
d_{1} \equiv-\left(\bar{\beta}_{1} B_{1}+1\right) a-1-\bar{\beta}_{1} \delta_{1} /\left(\bar{\beta}_{1} B_{1}+1\right) b
$$

$$
d_{2} \equiv \bar{\beta}_{1} C K /\left(\bar{\beta}_{1} B_{1}+1\right) b(1-\lambda)
$$

This is a differential equation whose particular integral is found by setting $\ddot{\Pi}^{1}=\dot{\Pi}^{1}=0$. Eq. (7) implies that at this point, $\Pi^{1}=\bar{\Pi}$. To obtain the trajectory we solve the quadratic equation $z^{2}+d_{1} z+d_{2}=0$, i.e.

$$
z=-d_{1} \pm \sqrt{\frac{d_{1}^{2}-4 d_{2}}{2}}
$$

The system is stable if both roots are negative. We have three cases.

1) If $d_{1}^{2}>4 d_{2}$ the roots $\left(z_{1}, z_{2}\right)$ are real numbers $z_{1} \neq z_{2}$. Stability requires $d_{2}<0$. This condition never holds because $d_{2}$ is positive. Thus, the system is unstable and the inflation rate diverges from the stationary equilibrium.

2) If $d_{1}^{2}=4 d_{2}$, both roots take the same value, $z_{1}=z_{2}=-d_{1} / 2$. Stability requires $d_{1}>0$, i.e., $a<1+\bar{\beta}_{1} \delta_{1} / \bar{\beta}_{1} B_{1}+1$.

3) If $d_{1}^{2}<4 d_{2}$, both roots are imaginary. For this case to arise, the competitive effect (i.e. CK) has to be large. In this circumstance the system describes a cyclic path. For equilibrium to be stable, i.e. for diminishing amplitude in cycle, we need $d_{1}>0$, i.e., $a<1+\bar{\beta}_{1} \delta_{1} / \bar{\beta}_{1} B_{1}+1$. 
If we have only the competitive effect $\left(\delta_{1}=\delta_{2}=B_{1}=B_{2}=0\right)$, the sufficient condition for convergence is $a<1$. If $a=1$ the trajectory is an orbit. Under no fiscal policy $\left(\delta_{1}=\delta_{2}=0\right)$, convergence occurs if $a<1 /$ $\bar{\beta}_{1} B_{1}+1$. In both cases stability requires some myopia in the perception of changes in inflation (if expectations are quickly revised $a \approx 1$ ). If fiscal authorities choose $\delta_{1}$ such that $d_{1}^{2} \leq 4 d_{2}$ and $a<1+\bar{\beta}_{1} \delta_{1} /$ $\bar{\beta}_{1} B_{1}+1$, inflation converges to stationary equilibrium. This highlights the importance of fiscal policy in order to attain convergence. Notice that the definition of average inflation implies that under instability two countries evolve in opposite directions: inflation increases in one country and decreases in the other.

\section{Conclusion}

In this note we have studied the effect of trade among country members on the stability of a currency area. In our model a large competitive effect is necessary but not sufficient for convergence. In absence of fiscal policy, stability also requires expectations not rational at the steady state $(a<1)$. If this condition fails, fiscal policy becomes necessary for convergence. Perhaps our model can shed light on the performance of some economies of EMU in recent years, particularly Germany, France and Spain. Needless to say, the robustness of our conclusions must be checked in more general models.

\section{References}

Alesina, A., Blanchard, O., Galí, J., Giavazzi, F., Uhlig, H., 2001. Defining a macroeconomic framework for the Euro area. Monitoring the European Central Bank 3.

Friedman, M., 1953. Essays in Positive Economics. University of Chicago Press, Chicago.

Kawai, M., 1987. Optimum currency areas. The New Palgrave: A Dictionary of Economics. Stockton Press, Nueva York, pp. $740-742$.

Kenen, P.B., 1969. The theory of optimum currency areas: an eclectic view. In: Mundell, R.A., Swoboda, A.K. (Eds.), Monetary

Problems of the International Economy. University of Chicago Press, Chicago, pp. 41-60.

McKinnon, R., 1963. Optimum currency areas. American Economic Review 53 (4), 717-725.

Mundell, R.A., 1961. A theory of optimum currency areas. American Economic Review 51 (4), 657-665.

Tobin, J., 1975. Keynesian models of recession and depression. American Economic Review 65 (2), 195-202. 